\title{
Importance of independent clinical research in medicine: difficulties and recommendations
}

Oscar Arrieta, Carlos Campillo, Rubén Burgos, Miguel Ángel Celis, Manuel De la Llata, Judith Domínguez, José Halabe, Sergio Islas, Luis Jasso*, Alberto Lifshitz, Mucio Moreno, Ricardo Plancarte, Alejandro Reyes-Sánchez, Guillermo J. Ruiz-Argüelles, Antonio Soda, Emma Verástegui and Julio Sotelo

Academia Nacional de Medicina, Comité de Ética y Transparencia en la Relación Médico-Industria, Ciudad de México, Mexico

\begin{abstract}
Clinical research is the most important tool for the identification of diagnostic and therapeutic strategies that derive in higher efficacy and safety. Despite its significance, successful implementation of clinical research faces numerous difficulties, with one of the most relevant being limited availability of resources for the performance of independent clinical trials. Generally, the pharmaceutical industry absorbs the costs associated with most clinical trials; however, this can generate dissociation between subjects of interest and health priorities when economic interest is the main driver of these protocols. In addition to the relevant role played by the pharmaceutical industry, it is important that government agencies favor adequate conditions, both in economic and regulatory aspects, for the implementation of independent clinical research that addresses subjects of medical and therapeutic interest, even if it does not generate corporate economic benefits.
\end{abstract}

KEY WORDS: Independent clinical research. Pharmaceutical companies. Research and development. Regulation.

Clinical research is an essential part of the mechanisms that determine medical- therapeutic algorithms; many of them use knowledge generated through medical research that is independent of industry-sponsored research for the creation of clinical practice guidelines. ${ }^{1,2}$ Despite these advantages, clinical research faces significant economic limitations due to the lack of available resources granted by government agencies. The financial responsibility for the conduction of research clinical studies usually falls in the pharmaceutical industry and therefore it shows several limitations: the pharmaceutical industry usually sponsors studies with a financial interest, not so others related to therapeutic validity or new therapeutic approaches of drugs whose patent has expired (e.g. metformin, nitroglycerin, chloroquine, etc.); therefore, the opportunity to carry out novel, high-impact projects with already-known molecules is lost. In addition, many clinical trials have the purpose to evaluate therapeutic behaviors and not a specific medication, such as studies that assess types of radical or conservative treatment in different clinical settings, as it occurs in cancer patients.

The cost for developing a new drug is enormous, and $60 \%$ of this budget is related to costs associated with clinical studies. For this reason, most clinical studies funded by the pharmaceutical industry have an economic interest in the approval and use of a drug. However, these costs also limit the capacity of independent research without financial interest. In most developing nations, governments assign suboptimal gross domestic product averages to scientific research, which significantly limits medical progress.
Date of reception:10-12-2018

Date of acceptance: $17-12-2018$

DOI: 10.24875/GMM.M19000275
Gac Med Mex. 2019;155:295-296

Contents available at PubMed www.gacetamedicademexico.com 
Elevated costs are the main reason whereby the industry finances most clinical trials. The industry has greater availability of resources to finance budgets, as well as a wide availability of administrative staff to take care of the paperwork required to achieve approval and adequate follow-up of study protocols.

There is a deficit of experienced clinical researchers to implement therapeutic trials. Clinical research is mostly carried out in public health institutions; independent clinical trials represent a significant workload increase without associated remuneration for the doctor-researcher. In addition to clinical trials follow-up, investigators must also prepare documents and reviews for ethics and research committees, and observe regulatory and even legal aspects. In contrast, industry-funded clinical trials have organizational support to hire staff that prepare documents and take care of obtaining the corresponding approvals. However, researchers hired by the industry for the conduction of clinical trials frequently act as its employees, rather than as independent researchers.

\section{Recommendations}

The Committee of Ethics and Transparency in the Physician-Industry Relationship (CETREMI - Comité de Ética y Transparencia en la Relación Médico-Industria) of the National Academy of Medicine recommends government institutions and non-profit organizations to support the development of independent clinical research protocols, with the following proposals:

1. The support granted by the government for clinical research, without a commercial interest for the industry, is remarkably limited. It is imperative for the spectrum of clinical research to be complemented by independent studies that act as a counterweight and address priority issues that don not represent economic interests.

2. Improving the training of clinical researchers at universities by incorporating to the medical undergraduate plans areas that offer early and comprehensive training in clinical research methodology, biostatistics and bioethics throughout the educational curriculum.

3. Improving the plan of stimuli offered to researchers to carry out independent initiative projects within health institutions.

4. Promoting and implementing pharmaceutical industry solidary support for independent clinical trials that are of medical interest, even with drugs with expired patents.

5. Categorizing, analyzing and adequately mitigating risks associated with research in order to reduce pharmacological trials costs.

6. Supporting the creation of cooperative groups that seek to address in a multidisciplinary and independent way relevant health problems in favor of the development of translational medicine.

7. Making more efficient and expeditious those administrative and regulatory processes that allow clinical trials adequate and safe implementation, including ethics and research committees, as well as availability of expert staff that collaborates with researchers in regulatory processes to implement independent clinical research protocols.

\section{References}

1. What is the purpose of medical research? Lancet. 2013;381:347.

2. Lechleiter J. Pharmaceutical companies sponsor clinical trials, but don't directly conduct them. Forbes 2015 Jul 21. 